MATEC Web of Conferences 11,01040 (2014)

DOI: $10.1051 /$ matecconf / 20141101040

C Owned by the authors, published by EDP Sciences, 2014

\title{
Les performances mécaniques et thermiques d'un béton léger à base de déchets industriels solides et de granulats de bois
}

\author{
Benmalek Mohamed Larbi ${ }^{1}$ et Bederina Madani ${ }^{2}$ \\ ${ }^{1}$ Université du 8 mai 1945, Faculté des Sciences et de la technologie, Département de Génie Civil et Hydraulique, Guelma, \\ Algérie et Laboratoire de Génie Civil et Hydraulique (LGCH). \\ ${ }^{2}$ Université Amar Thelidji, Faculté des Sciences et de la technologie, Département de Génie Civil, Laghouat, Algérie.
}

\begin{abstract}
L'industrie des granulats et celle du travail de la pierre génèrent des quantités importantes de déchets solides plus ou moins fins. Ces fines constituent bien souvent un problème pour l'environnement. Elles se présentent en effet, dans le cas d'une extraction à sec, sous forme de poussières aisément transportées par les courants d'air entraînant une pollution physique et esthétique de l'environnement. D'un autre côté, les boues provenant des bassins de décantation autour des carrières d'exploitation des granulats, ont longtemps été accumulés dans la nature et forment actuellement des montagnes de résidus solides inexploités.

Afin d'étudier les potentialités de valorisation de ces déchets industriels, le choix a été porté vers la conception de bétons d'isolation dont l'allégement a été obtenu par l'incorporation de granulats de bois de 3 à $8 \mathrm{~mm}$ de dimension dans une matrice constituée de ciment portland et d'une fine minérale. La technique utilisée est peu consommatrice d'énergie, peu coûteuse et non polluante. Cinq fines de différentes natures minéralogiques et de différentes (esthétiques) couleurs ont été sélectionnées pour cette étude.

Dans cette communication, les auteurs présentent les performances mécaniques et thermiques des matériaux élaborés : les bétons d'isolation et leurs matrices seules.

Les résultats expérimentaux montrent qu'à densité égale, les matériaux élaborés sont mécaniquement et thermiquement compétitifs. Ils sont intéressants du point de vue confort et stockage thermiques.
\end{abstract}

\section{Introduction}

Cinq fines de différentes natures minéralogiques provenant de l'industrie d'exploitation de carrières ont été traitées et transformées en bétons légers d'isolation. Le mode d'allégement choisi est l'incorporation de granulats de bois traités dans une matrice constituée d'une fine minérale et de ciment portland. Les bétons légers ainsi élaborés constituent des matériaux compétitifs non seulement aux bétons légers traditionnels à base d'argile expansée, de schiste expansé, de vermiculite, de perlite ou encore de cendres volantes mais aussi aux bétons légers récemment étudiées contenant des granulats de liège [1], des balles de riz [2], des cendres de balles de riz [3] ou des copeaux de bois [4].

La technologie employée pour développer ces nouveaux matériaux de construction est peu coûteuse. La quantité de stabilisant (ciment) utilisée est faible et le matériau élaboré fini ne nécessite aucun traitement thermique supplémentaire avant utilisation.

Les bétons légers élaborés sont manifestement destinés à la confection d'éléments de constructions thermiquement et phoniquement isolants, par conséquent la mesure de leurs performances thermiques est une nécessité incontournable.

Dans cette communication, les auteurs présentent la mesure des 3 plus importants paramètres thermiques, ce sont: la conductivité thermique, $\lambda$; la diffusivité thermique, $a$; et la chaleur spécifique volumique, $C$. Les résultats sont évalués en fonction de la densité à sec mesurée, des conditions extrêmes d'usage et du critère de confort thermique. Ils sont alors comparés aux paramètres thermiques de différents matériaux légers de construction.

Les résultats des mesures des résistances mécaniques sont également présentés et analysés.

\section{Matériaux utilisés}

\subsection{Matière première}

Les fines minérales ont été collectées en Bretagne (Nord-Ouest de la France); leurs provenances et leurs aspects sont reportés dans le tableau 1.

Pour cette étude, les résidus recueillis sous forme de boues ont dû subir un traitement afin de mieux maîtriser les teneurs en eau : séchage dans une étuve ventilée à $105^{\circ} \mathrm{C}$ pendant 24 heures, broyage durant une période de 3 heures, tamisage par vibration électrique au tamis de module $32(1.25 \mathrm{~mm})$ afin d'éliminer les différentes impuretés qui ont pu polluer le bassin de décantation et enfin, stockage en salle sèche $\left(20^{\circ} \mathrm{C}\right.$ et $50 \%$ d'humidité relative). 
Tableau 1. Provenance et aspect des fines.

\begin{tabular}{|l|l|l|}
\hline Fines & \multicolumn{1}{|c|}{ Provenance } & \multicolumn{1}{|c|}{ Aspect initial } \\
\hline Granite & $\begin{array}{l}\text { Déchets des travaux de sciage } \\
\text { et de finition de surfaces de la } \\
\text { roche massive. Recueillis dans } \\
\text { le bassin de décantation de la } \\
\text { carrière de Baillé (Morbihan). }\end{array}$ & $\begin{array}{l}\text { Boues lisses au } \\
\text { toucher, de couleur } \\
\text { grisâtre. }\end{array}$ \\
\hline Grès & $\begin{array}{l}\text { Résidus d'exploitation de } \\
\text { granulats par concassage. } \\
\text { Extraits après concassage, par } \\
\text { lavage intense à l'eau. } \\
\text { Recueillis dans le bassin de } \\
\text { décantation de la carrière de } \\
\text { Fréhel (Côte d'Armor). }\end{array}$ & $\begin{array}{l}\text { Boues quartzitiques } \\
\text { de couleur rosâtre. }\end{array}$ \\
\hline Schiste & $\begin{array}{l}\text { Résidus de concassage de la } \\
\text { roche massive. Le procédé de } \\
\text { séparation des granulats } \\
\text { concassés est un procédé à sec. } \\
\text { Recueillis de la carrière des } \\
\text { Lacs (Ille et Vilaine) }\end{array}$ & $\begin{array}{l}\text { Farines très fines et } \\
\text { relativement } \\
\text { homométriques, de } \\
\text { couleur gris } \\
\text { verdâtre. }\end{array}$ \\
\hline Argile 1 & $\begin{array}{l}\text { Résultent de l'exploitation de } \\
\text { placages de sables marins } \\
\text { fossiles. Résidus de lavage des } \\
\text { granulats. Recueillis dans le } \\
\text { bassin de décantation du Rheu } \\
\text { (Ille et Vilaine) }\end{array}$ & $\begin{array}{l}\text { Boues de couleur } \\
\text { brun clair contenant } \\
\text { une portion } \\
\text { d'environ 5 à 10 \% } \\
\text { de quartz. }\end{array}$ \\
\hline Argile 2 & $\begin{array}{l}\text { Résultent de l'exploitation } \\
\text { d'une carrière de sable } \\
\text { alluvionnaire. Recueillis dans } \\
\text { carrière de Mohon (Morbihan). }\end{array}$ & $\begin{array}{l}\text { Boues de couleur } \\
\text { jaunâtre, rugueuses, } \\
\text { contenant une } \\
\text { proportion } \\
\text { importante de } \\
\text { quartz. }\end{array}$ \\
\hline
\end{tabular}

Les fines minérales traitées ont été caractérisées par leurs distributions granulométriques, leurs masses volumiques apparentes et leurs conductivités thermiques intrinsèques (tableau 2).

Tableau 2. Masse volumique et conductivité thermique des fines minérales.

\begin{tabular}{|l|c|c|c|}
\hline $\begin{array}{l}\text { Nature } \\
\text { des fines }\end{array}$ & $\begin{array}{c}\text { Limites granulaires } \\
(\mu \mathrm{m})\end{array}$ & $\begin{array}{c}\text { Masse volumique } \\
\left(\mathrm{kg} / \mathrm{m}^{3}\right)\end{array}$ & $\begin{array}{c}\lambda_{\text {fine }} \\
(\mathrm{W} / \mathrm{mK})\end{array}$ \\
\hline Granite & $1-40$ & 2610 & 2.58 \\
Grès & $0.04-150$ & 2640 & 4.72 \\
Schiste & $1-40$ & 2800 & 3.76 \\
Argile 1 & $0.04-50$ & 2600 & 2.35 \\
Argile 2 & $0.04-100$ & 2620 & 3.61 \\
\hline
\end{tabular}

Le ciment, assurant la stabilisation est de type CPACEM I 52.5, il est conforme à la norme européenne (EN $1961)$.

L'eau de gâchage est une eau potable du réseau, de $\mathrm{pH}$ $\sim$, conforme à la norme NF P15-401.

Les granulats de bois utilisés sont commercialisés "Agrelith C", ils sont d'essence épicéa et ont des dimensions 3-8 mm. Leur traitement (Brevet Français $\mathrm{N}^{\circ}$ 8102941) favorise la limitation des variations dimensionnelles des bétons élaborés. Ils présentent une structure poreuse tubulaire (fig. 1) qui explique leur faible masse volumique à sec $\left(\rho_{\mathrm{S}}=255 \mathrm{~kg} / \mathrm{m}^{3}\right)$.

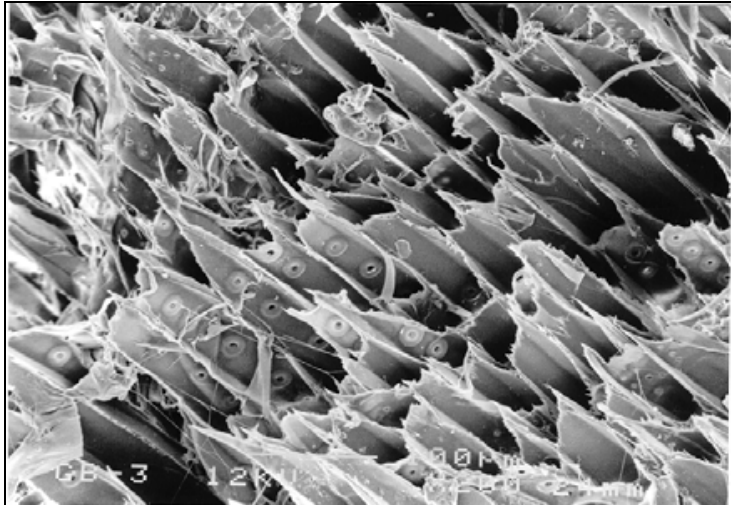

Fig. 1. Aspect général au MEB du granulat de bois traité (x 200)

\subsection{Elaboration des bétons d'étude}

Les matériaux composites élaborés dans ce travail ont tous la même répartition pondérale à sec. Seule la nature des fines diffère. Les proportions massiques sont: $25 \%$ de ciment $(\mathrm{C})+50 \%$ de fines minérales $(\mathrm{F})+25 \%$ de bois (B). Soit un rapport massique constant $\mathrm{F} / \mathrm{C}=2$. Le même rapport a été fixé pour la confection des matériaux sans bois (matrices).

La quantité de bois a été déterminée par Bouguerra [5] pour obtenir des densités correspondant aux valeurs cibles du programme Européen FOREST, selon Pimienta [6]. La quantité d'eau ajoutée a été déterminée à l'aide de la formule générale empirique suivante [7]:

$\mathrm{E}=\mathrm{W}_{\mathrm{c}} \mathrm{C}+\mathrm{W}_{\mathrm{f}} \mathrm{F}+\mathrm{W}_{\mathrm{b}^{\mathrm{B}}}$

où $\mathrm{W}_{\mathrm{c}}$ est un coefficient permettant d'obtenir une pâte de ciment de consistance normale(norme EN 196 - 3). $\mathrm{W}_{\mathrm{c}}=$ 0.35 selon Moslemi [8] et Al Rim [7].

$\mathrm{W}_{\mathrm{f}}$ est un coefficient relatif aux fines étudiées permettant d'obtenir une pâte fine-eau de consistance normale également. Nous avons, nous mêmes, obtenu:

$\mathrm{W}_{\mathrm{f}}=0.45$ pour les fines de granite, grès et schiste.

$\mathrm{W}_{\mathrm{f}}=0.70$ pour les fines d'argile 1 et d'argile 2 .

$\mathrm{W}_{\mathrm{b}}$ est un coefficient relatif aux granulats de bois traités, il correspond à l'absorption d'eau à refus des granulats de bois. $\mathrm{W}_{\mathrm{b}}=0.80$ selon [5].

Soit: $\mathrm{E}=0.35 \mathrm{C}+(0.45$ ou 0.70$) \mathrm{F}+0.80 \mathrm{~B}$

Le tableau 3 indique les quantités utilisées pour $1 \mathrm{~m}^{3}$ de béton de bois. La masse volumique théorique du matériau composite a été prise égale à $800 \mathrm{~kg} / \mathrm{m}^{3}$.

Les constituants utilisés dans la préparation des mélanges sont préalablement séchés afin de mieux contrôler l'eau de gâchage.

Concernant la préparation, un premier malaxage à sec est indispensable pour bien homogénéiser le mélange : les fines et le ciment sont introduits dans un malaxeur à mortier normalisé à axe vertical (norme EN. 196-1) et mélangés pendant 3 minutes à vitesse lente. 
Tableau 3. Proportions massiques théoriques des constituants pour la confection de $1 \mathrm{~m}^{3}$ de matériau

\begin{tabular}{|c|c|c|c|c|c|c|}
\hline $\begin{array}{l}\text { Fine } \\
\text { employée }\end{array}$ & Matériau & Mélange & $\begin{array}{l}\text { Ciment } \\
(\mathrm{kg})\end{array}$ & $\begin{array}{c}\text { Fine minérale } \\
(\mathrm{kg})\end{array}$ & $\begin{array}{c}\text { Granulat de bois } \\
(\mathrm{kg})\end{array}$ & $\begin{array}{l}\text { Eau } \\
(\mathrm{kg})\end{array}$ \\
\hline Granite & $\begin{array}{l}\text { Matrice } \\
\text { Composite }\end{array}$ & $\begin{array}{l}\text { M1 } \\
\text { M2 }\end{array}$ & $\begin{array}{l}267 \\
200\end{array}$ & $\begin{array}{l}533 \\
400 \\
\end{array}$ & 200 & $\begin{array}{l}280 \\
410\end{array}$ \\
\hline Grès & $\begin{array}{l}\text { Matrice } \\
\text { Composite }\end{array}$ & $\begin{array}{l}\text { M3 } \\
\text { M4 }\end{array}$ & $\begin{array}{l}267 \\
200 \\
\end{array}$ & $\begin{array}{l}533 \\
400 \\
\end{array}$ & $\begin{array}{c}- \\
200 \\
\end{array}$ & $\begin{array}{l}280 \\
410 \\
\end{array}$ \\
\hline Schiste & $\begin{array}{l}\text { Matrice } \\
\text { Composite }\end{array}$ & $\begin{array}{l}\text { M5 } \\
\text { M6 }\end{array}$ & $\begin{array}{l}267 \\
200 \\
\end{array}$ & $\begin{array}{l}533 \\
400 \\
\end{array}$ & $\begin{array}{c}- \\
200 \\
\end{array}$ & $\begin{array}{l}280 \\
410 \\
\end{array}$ \\
\hline Argile 1 & $\begin{array}{l}\text { Matrice } \\
\text { Composite }\end{array}$ & $\begin{array}{l}\text { M7 } \\
\text { M8 } \\
\end{array}$ & $\begin{array}{l}267 \\
200 \\
\end{array}$ & $\begin{array}{l}533 \\
400 \\
\end{array}$ & $\begin{array}{c}- \\
200 \\
\end{array}$ & $\begin{array}{l}333 \\
510 \\
\end{array}$ \\
\hline Argile 2 & $\begin{array}{l}\text { Matrice } \\
\text { Composite }\end{array}$ & $\begin{array}{l}\text { M9 } \\
\text { M10 }\end{array}$ & $\begin{array}{l}267 \\
200\end{array}$ & $\begin{array}{l}533 \\
400\end{array}$ & $\begin{array}{c}- \\
200\end{array}$ & $\begin{array}{l}333 \\
510\end{array}$ \\
\hline
\end{tabular}

Le mélange étant parfaitement homogène, on ajoute les granulats de bois en conservant le malaxage à vitesse lente pendant 3 autres minutes. L'eau de gâchage est ensuite graduellement versée pendant le malaxage. L'homogénéisation est assurée par un malaxage à vitesse lente pendant une durée de 3 minutes puis à vitesse rapide pendant 1 minute. Le mélange homogène obtenu est alors mis en moule, avec soin, par coulage et léger tassage afin de ne pas occlure d'air dans la pâte fraîche. L'excès de béton est enlevé par arasement. Les moules remplis sont conservés en salle à hygrométrie et température contrôlées $\left(20^{\circ} \mathrm{C}\right.$ et $90 \%$ d'humidité relative). Le démoulage se fait un jour après la fabrication et les éprouvettes sont ensuite placées dans la salle de conservation, jusqu'à réalisation des essais.

Dans le tableau 4 qui suit, sont reportées, en $\mathrm{kg} / \mathrm{m}^{3}$, les masses volumiques à sec des matériaux élaborés après 28 jours, elles ont été déterminées par pesée et mesure des dimensions d'éprouvettes cubiques de $10 \mathrm{~cm}$ d'arête.

Tableau 4. Masses volumiques à sec à 28 jours $\left(\mathrm{kg} / \mathrm{m}^{3}\right)$

\begin{tabular}{|l|c|c|}
\hline $\begin{array}{l}\text { Fine } \\
\text { minérale }\end{array}$ & Matrice & Composite \\
\hline Granite & 1460 & 900 \\
Grès & 1590 & 860 \\
Schiste & 1500 & 960 \\
Argile 1 & 1270 & 810 \\
Argile 2 & 1290 & 810 \\
\hline
\end{tabular}

Avec la composition adoptée, les masses volumiques des composites varient entre 810 et $960 \mathrm{~kg} / \mathrm{m}^{3}$ pour une même teneur en granulats de bois. Le béton de bois à base de fines schisteuses présente toutefois une valeur plus importante qui serait liée à sa plus fine granulométrie, celle du granite a une étendue granulaire semblable.

Les valeurs des masses volumiques sont proches. Notons que tous les matériaux élaborés sont des matériaux légers $\left(\mathrm{M}_{\mathrm{v}}<1800 \mathrm{~kg} / \mathrm{m}^{3}\right)$.

En dehors de la masse volumique du béton de schiste, les autres entrent bien dans la fourchette des cibles du programme européen FOREST $\left(600-900 \mathrm{~kg} / \mathrm{m}^{3}\right)$. Ces limites s'appuient sur un compromis entre les propriétés thermiques et mécaniques des bétons de bois à matrice cimentaire. Au delà de $900 \mathrm{~kg} / \mathrm{m}^{3}$, l'isolation thermique diminue considérablement et en deçà de $600 \mathrm{~kg} / \mathrm{m}^{3}$, c'est la résistance mécanique qui diminue nettement [6].

\section{Techniques expérimentales}

\subsection{Mesure de la conductivité thermique}

Les mesures de conductivité thermique ont été effectués dans une enceinte climatique de type Heraeus VTK 150 à des températures de $10,20,40$ et $60^{\circ} \mathrm{C}$ et à différentes teneurs en eau. La technique de mesure choisie est celle de la sonde monotige en raison du caractère ponctuel de la mesure. Le temps de la manipulation est très court et les élévations de température faibles, limitant en cela les phénomènes de thermo-migrations à l'intérieur du matériau.

Cette méthode transitoire de mesure a fait l'objet de plusieurs publications, notamment celles de Perrin [9] et Laurent $[10,11]$. Son principe repose sur la mesure de l'élévation de la température dans une tige métallique cylindrique de résistance connue introduite au sein du matériau à étudier. La température de surface de la sonde est mesurée à l'aide d'un thermocouple dont la soudure est située à mi-hauteur. Avec cette sonde, il est possible de mesurer la conductivité thermique des matériaux granulaires, des matériaux suffisamment pâteux et des matériaux consolidés.

La conductivité thermique du matériau est déterminée par régression linéaire à partir de la relation logarithmique (3) qui suit:

$$
\Delta T(t)=\frac{Q}{4 \pi \lambda} \operatorname{Ln}(t)+A
$$

$\Delta \mathrm{T}(\mathrm{t})$ : Variation de température dans le temps $(\mathrm{K})$

Q: Flux thermique dissipé par unité de longueur de l'élément chauffant $(\mathrm{W} / \mathrm{m})$

$\lambda$ : Conductivité thermique du matériau à étudier

A: Constante dépendant de divers paramètres de la sonde (type d'élément chauffant, rayon de l'élément, type de thermocouple...).

\subsection{Mesure simultanée de la chaleur spécifique volumique et de la diffusivité thermique}

La technique utilisée pour réaliser les mesures de ces paramètres thermiques, à l'état sec et à l'état saturé, est la technique TPS (Transient Plane Source) développée par Gustaffson [12]. 


\section{Résultats et analyse}

\subsection{Conductivité thermique}

Le tableau 5 regroupe les résultats expérimentaux obtenus sur les matériaux élaborés dans les états sec et saturé en eau, à température ambiante $\left(\sim 20^{\circ} \mathrm{C}\right)$.

Les bétons de bois élaborés ont un grand pouvoir d'isolation thermique, les valeurs de $\lambda$ sont inférieures à $0.2 \mathrm{~W} / \mathrm{mK}$ quelle que soit la matrice. Ils peuvent être considérés, de ce point de vue, comme matériaux isolants de type II dans la classification fonctionnelle de la RILEM [13]. Les composites à base des fines non argileuses sont les plus thermiquement conducteurs.

On peut noter également que les granulats de bois réduisent, dans tous les cas, la conductivité thermique dans un rapport $\lambda_{\mathrm{ms}} / \lambda_{\mathrm{cs}}$ supérieur à 2 : de 2 dans le cas du béton de bois à base de fines de granite à 2.6 environ dans celui du béton de bois à base de fines de grès. Il en ressort que l'influence du bois est prépondérante dans la conductivité thermique. Les granulats de bois interviennent dans la modification de la tortuosité notamment.

La conductivité thermique des fines minérales qui les composent et leurs masses volumiques (tableau. 2) ne peuvent pas, à elles seules, expliquer ces résultats. En effet, les 2 fines argileuses ont des masses volumiques voisines et des conductivité thermiques très différentes. Les densités sèches des matériaux élaborés influent directement sur leurs conductivités thermiques, la proportionnalité est manifeste si l'on observe les valeurs des 2 paramètres présentés dans le tableau 5 .

Le tableau 6 présente les valeurs de $\lambda$ de quelques bétons de bois et matériaux légers usuels. On voit qu'à densité égale, les matériaux élaborés sont thermiquement compétitifs.

L'évolution de la conductivité thermique en fonction de la température à des teneurs en eau données, comprises entre l'état sec et l'état saturé, a également été étudiée sur les matériaux élaborés [16].

Il a été montré qu'aux températures supérieures à $40^{\circ} \mathrm{C}$ et dès que les matériaux poreux élaborés contiennent des quantités d'eau relativement importantes ( $w \sim 20-30 \%$ ), le phénomène d'évaporation- condensation apparaît dans l'espace poral des matériaux; ceci est traduit par la disparition de la linéarité dans la courbe d'évolution.

\subsection{Capacité calorifique volumique}

Ce paramètre thermique a été mesuré à température ambiante par la méthode TPS sur l'ensemble des matrices et des composites élaborés. Les états de saturation considérés sont les états extrêmes: sec et saturé par immersion dans l'eau.

Les valeurs de mesures présentées dans le tableau 7 constituent les moyennes sur 3 mesures.
La capacité calorifique volumique est un paramètre indiquant la quantité de chaleur nécessaire pour produire une élévation de température de 1 degré dans un volume de $1 \mathrm{~m}^{3}$. Il existe peu de données bibliographiques concernant les matériaux de construction et en particulier les bétons légers. Dans ce travail, nous avons mesuré ce paramètre thermique sur les matrices et les composites élaborés afin d'apprécier l'aptitude de ces matériaux à emmagasiner la chaleur et par conséquent pour apprécier leurs apports dans le confort thermique. De plus, les mesures ont été réalisées seulement à température ambiante $\left(\sim 20^{\circ} \mathrm{C}\right)$ car ce paramètre est très peu sensible à la température [5] pour les bétons de bois à matrice argileuse.

Les valeurs des capacités calorifiques volumiques à sec et à saturation sont regroupées dans le tableau 7. On voit que ce paramètre thermique est fortement influencé par la teneur en eau des matériaux. Par ailleurs, la loi d'additivité des enthalpies (équation 4) est assez bien validée dans le cas des matériaux élaborés (tableau 8). En effet, les écarts maximums entre les valeurs mesurées $\mathrm{C}_{\theta}$ et les valeurs calculées sont $7.4 \%$ pour les bétons de bois et $5.1 \%$ pour les matrices.

$$
\begin{gathered}
\mathrm{C}_{\theta}=\mathrm{C}_{\mathrm{sec}}+\theta \times \mathrm{C}_{\text {eau }}\left(\mathrm{J} / \mathrm{m}^{3} \cdot \mathrm{K}\right) \\
\mathrm{C}_{\theta}=\mathrm{C}_{\mathrm{sec}}+4.18 \times 10^{6} \theta\left(\mathrm{J} / \mathrm{m}^{3} \cdot \mathrm{K}\right)
\end{gathered}
$$

$\theta$ : Teneur volumique en eau du matériau étudié.

$\theta=\mathrm{d} \times \mathrm{w}(\mathrm{d}:$ densité sèche et $\mathrm{w}$ : teneur massique en eau)

On peut constater que la relation entre ce paramètre et la densité des matériaux élaborés n'est pas très bien établie. Pour l'exemple, le béton de bois à base de granite a une densité sèche $(\mathrm{d}=0.90)$ située entre celle du béton à base de grès $(\mathrm{d}=0.86)$ et celle du béton à base de schiste $(\mathrm{d}=0.96)$ mais une capacité calorifique à $\sec \left(\mathrm{C}_{\mathrm{sec}}\right.$ $\left.=1.02 \mathrm{MJ} / \mathrm{m}^{3} . \mathrm{K}\right)$ inférieure à celle des deux autres composites (respectivement 1.16 et $1.10 \mathrm{MJ} / \mathrm{m}^{3} . \mathrm{K}$ ).

Notons également que les capacités calorifiques à sec des matrices sont toutes supérieures à celles des composites à cause de la présence du bois.

\subsection{Diffusivité thermique}

Les valeurs mesurées de ce paramètre, reportées dans le tableau 7 indiquent que la diffusivité thermique ne dépend que très peu de la teneur en eau des composites élaborés. Pour les matrices l'influence de la teneur en eau est plus importante. Morabito [17] dans son étude sur des bétons de diverses natures et de différentes densités, a fait les mêmes observations pour des faibles teneurs en eau.

La figure 2 montre l'évolution de la diffusivité thermique avec la densité. On peut constater qu'à une densité de matériau faible correspond une diffusivité thermique faible. La complexité du phénomène de diffusion thermique dans les matériaux hétérogènes tels que les matériaux de construction n'autorise pas, à ce niveau de notre étude, l'établissement d'une loi générale reliant ces 2 paramètres. 
Tableau 5. Conductivités thermiques des matériaux élaborés (indices: $\mathrm{m}$, matrice; c, composite; $\mathrm{s}$, état sec; sat, état saturé)

\begin{tabular}{|l|c|c|c|c|c|c|c|c|}
\hline \multirow{3}{*}{ Fine employée } & \multicolumn{3}{|c|}{ MATRICES } & \multicolumn{3}{c|}{ COMPOSITES } & \multirow{2}{*}{$\lambda_{\text {ms }} / \lambda_{\text {cs }}$} & \multirow{2}{*}{$\lambda_{\text {msat }} / \lambda_{\text {csat }}$} \\
\cline { 2 - 7 } & $\begin{array}{c}\mathrm{d}_{\text {sec }} \\
\left(\mathrm{kg} / \mathrm{m}^{3}\right)\end{array}$ & $\begin{array}{c}\lambda_{\text {ms }} \\
(\mathrm{W} / \mathrm{mK})\end{array}$ & $\begin{array}{c}\lambda_{\text {msat }} \\
(\mathrm{W} / \mathrm{mK})\end{array}$ & $\begin{array}{c}\mathrm{d}_{\text {sec }} \\
\left(\mathrm{kg} / \mathrm{m}^{3}\right)\end{array}$ & $\begin{array}{c}\lambda_{\text {cs }} \\
(\mathrm{W} / \mathrm{mK})\end{array}$ & $\begin{array}{c}\lambda_{\text {csat }} \\
(\mathrm{W} / \mathrm{mK})\end{array}$ & & \\
\hline Granite & 1460 & 0.359 & 0.860 & 900 & 0.178 & 0.611 & 2.02 & 1.41 \\
Grès & 1590 & 0.457 & 0.950 & 860 & 0.175 & 0.556 & 2.61 & 1.71 \\
Schiste & 1500 & 0.366 & 0.894 & 960 & 0.190 & 0.570 & 2.03 & 1.57 \\
Argile 1 & 1270 & 0.189 & 0.786 & 810 & 0.117 & 0.529 & 2.08 & 1.49 \\
Argile 2 & 1290 & 0.252 & 0.833 & 810 & 0.117 & 0.532 & 2.15 & 1.57 \\
\hline
\end{tabular}

Tableau 6. Conductivité thermique de quelques matériaux légers.

\begin{tabular}{|c|c|c|c|c|}
\hline Matériaux & $\begin{array}{l}\text { Granulats } \\
\text { de bois }\end{array}$ & Matrice & $\begin{array}{l}\text { Masse volumique } \\
\text { sèche }\left(\mathrm{Kg} / \mathrm{m}^{3}\right)\end{array}$ & $\begin{array}{c}\lambda_{\mathrm{sec}} \\
(\mathrm{W} / \mathrm{mK})\end{array}$ \\
\hline \multirow{4}{*}{ Bétons de bois usuels } & Agreslith $^{1}$ & Ciment & $500-700$ & $0.11-0.16$ \\
\hline & Granuland $^{1}$ & Ciment & 800 & 0.22 \\
\hline & & Ciment + sable & 700 & 0.15 \\
\hline & Isochanvre $^{2}$ & Chaux & 800 & 0.27 \\
\hline $\begin{array}{l}\text { Bétons de bois à } \\
\text { matrice argileuse }\end{array}$ & Agreslith & Ciment + argile & $\begin{array}{c}500 \\
1000\end{array}$ & $\begin{array}{l}0.13 \\
0.29\end{array}$ \\
\hline $\begin{array}{l}\text { Bétons de perlite ou } \\
\text { Vermiculite } 4\end{array}$ & - & - & $\begin{array}{c}400-600 \\
600-800\end{array}$ & $\begin{array}{l}0.24 \\
0.31\end{array}$ \\
\hline $\begin{array}{l}\text { Béton à granulats d'argile } \\
\text { expansée } 4\end{array}$ & - & - & $\begin{array}{c}600-800 \\
800-1000\end{array}$ & $\begin{array}{l}0.25 \\
0.33\end{array}$ \\
\hline Plâtre & - & - & 1060 & 0.36 \\
\hline Béton cellulaire autoclavé & - & - & 500 & 0.18 \\
\hline $\begin{array}{l}\text { Bétons de bois } \\
\text { minérales }\end{array}$ & Agreslith & $\begin{array}{l}\text { Ciment }+ \text { fines } \\
\text { minérales }\end{array}$ & $810-960$ & $0.117-0.190$ \\
\hline
\end{tabular}

${ }_{5}^{1}$ : P.Piementa et al, 1994 [6]; ${ }^{2}$ : P.Sionneau et al, 1994 [14]; ${ }^{3}$ : A.Bouguerra, 1997[5]; ${ }^{4}$ : Règles DTU Françaises Th-K77 [15]; ${ }^{5}$ : Présent travail

Tableau 7. Capacité calorifique volumique et diffusivité thermique des matériaux élaborés

\begin{tabular}{|l|c|c|c|c|c|}
\hline \multirow{2}{*}{$\begin{array}{l}\text { Fine minérale } \\
\text { employée }\end{array}$} & \multirow{2}{*}{ Densité } & $\mathrm{a}\left(10^{-7} \mathrm{~m}^{2} / \mathrm{s}\right)$ & $\mathrm{C}\left(\mathrm{MJ} / \mathrm{m}^{3} . \mathrm{K}\right)$ & $\mathrm{a}\left(10^{-7} \mathrm{~m}^{2} / \mathrm{s}\right)$ & $\mathrm{C}\left(\mathrm{MJ} / \mathrm{m}^{3} . \mathrm{K}\right)$ \\
\hline \multicolumn{7}{|c|}{ COMPOSITES } \\
\hline Granite & 0.90 & 2.43 & 1.02 & 2.50 & 2.61 \\
Grès & 0.86 & 2.20 & 1.16 & 2.27 & 2.76 \\
Schiste & 0.96 & 2.65 & 1.10 & 2.14 & 2.47 \\
Argile 1 & 0.81 & 2.00 & 1.27 & 2.15 & 3.19 \\
Argile 2 & 0.81 & 2.03 & 1.23 & 4.51 & 3.19 \\
\hline \multicolumn{7}{|c|}{ MATRICES } & 4.82 & 3.68 \\
\hline Granite & 1.46 & 3.28 & 1.60 & 4.66 & 3.94 \\
Grès & 1.59 & 3.49 & 1.80 & 4.40 & 3.93 \\
Schiste & 1.50 & 3.32 & 1.78 & 4.43 & \\
Argile 1 & 1.27 & 2.92 & 2.02 & 1.92 & \\
Argile 2 & 1.29 & 2.94 &
\end{tabular}

Tableau 8. Tableau comparatif des capacités calorifiques théoriques et expérimentales

\begin{tabular}{|c|c|c|c|c|c|c|c|}
\hline $\begin{array}{l}\text { Fine minérale } \\
\text { employée }\end{array}$ & d & $\begin{array}{l}\mathrm{W} \\
(\%)\end{array}$ & $\begin{array}{c}\theta \\
(\%) \\
\end{array}$ & $\begin{array}{c}\mathrm{C}_{\mathrm{sec}} \\
\mathrm{MJ} / \mathrm{m}^{3} \cdot \mathrm{K} \\
\end{array}$ & $\begin{array}{c}\mathrm{C}_{\theta} \\
\mathrm{MJ} / \mathrm{m}^{3} \cdot \mathrm{K} \\
\end{array}$ & $\begin{array}{l}\mathrm{C}_{\text {Өthéorique }} \\
\mathrm{MJ} / \mathrm{m}^{3} . \mathrm{K} \\
\end{array}$ & $\begin{array}{c}\text { Ecart/th } \\
(\%)\end{array}$ \\
\hline \multicolumn{8}{|c|}{ COMPOSITES } \\
\hline Granite & 0.90 & 45 & 41 & 1.02 & 2.61 & 2.73 & 4.40 \\
\hline Grès & 0.86 & 42 & 36 & 1.16 & 2.76 & 2.66 & -3.76 \\
\hline Schiste & 0.96 & 38 & 36 & 1.10 & 2.47 & 2.60 & 5.00 \\
\hline Argile 1 & 0.81 & 54 & 44 & 1.27 & 2.88 & 3.11 & 7.40 \\
\hline Argile 2 & 0.81 & 55 & 45 & 1.23 & 3.19 & 3.11 & -2.57 \\
\hline \multicolumn{8}{|c|}{ MATRICES } \\
\hline Granite & 1.46 & 26 & 38 & 1.60 & 3.04 & 3.19 & 4.70 \\
\hline Grès & 1.59 & 28 & 45 & 1.80 & 3.51 & 3.68 & 1.70 \\
\hline Schiste & 1.50 & 26 & 39 & 1.78 & 3.26 & 3.41 & 4.40 \\
\hline Argile 1 & 1.27 & 33 & 46 & 2.02 & 3.74 & 3.94 & 5.10 \\
\hline Argile 2 & 1.29 & 36 & 48 & 1.92 & 4.00 & 3.93 & -1.80 \\
\hline
\end{tabular}


Tableau 9. Paramètres thermiques de quelques matériaux légers

\begin{tabular}{|c|c|c|c|c|c|}
\hline Matériaux & $\rho: \mathrm{Kg} / \mathrm{m}^{3}$ & $\lambda: \mathrm{W} / \mathrm{mK}$ & $\mathrm{C}: \mathrm{MJ} / \mathrm{m}^{3} \cdot \mathrm{K}$ & a: $10^{-7} \mathrm{~m}^{2} / \mathrm{s}$ & c: J/kg.K \\
\hline Béton cellulaire autoclavé & 500 & 0.18 & 0.42 & 3.16 & 900 \\
\hline Plâtre sec & 1060 & 0.35 & 1.02 & 3.57 & 961 \\
\hline Plâtre saturé & 1440 & - & 1.55 & 3.16 & - \\
\hline $\begin{array}{l}\text { Béton argileux de copeaux de } \\
\text { bois } 1\end{array}$ & 1054 & 0.24 & 0.93 & 2.23 & 1008 \\
\hline $\begin{array}{l}\text { Béton argileux cellulaire non } \\
\text { autoclavé }^{2}\end{array}$ & $700-1500$ & $0.16-0.58$ & - & $2-2.80$ & $800-1000$ \\
\hline Béton d'argile expansé ${ }^{3}$ & 1520 & 0.64 & 0.54 & - & - \\
\hline $\begin{array}{l}\text { Béton argileux de granulats de } \\
\text { bois }{ }^{4}\end{array}$ & 1280 & 0.45 & 1.40 & 2.70 & 1100 \\
\hline $\begin{array}{l}\text { Matrice } \\
\qquad 30 \% \text { de } G B\end{array}$ & 820 & 0.20 & 1.00 & 2.25 & 1260 \\
\hline Bétons de fines ${ }^{5}$ & $810-960$ & $0.117-0.19$ & $2.47-3.19$ & $2.14-2.72$ & - \\
\hline
\end{tabular}

1: Al Rim [4]; 2 : Marmoret [18]; 3 : Morabito [17]; 4 : Bouguerra [5]; 5 : Présent travail

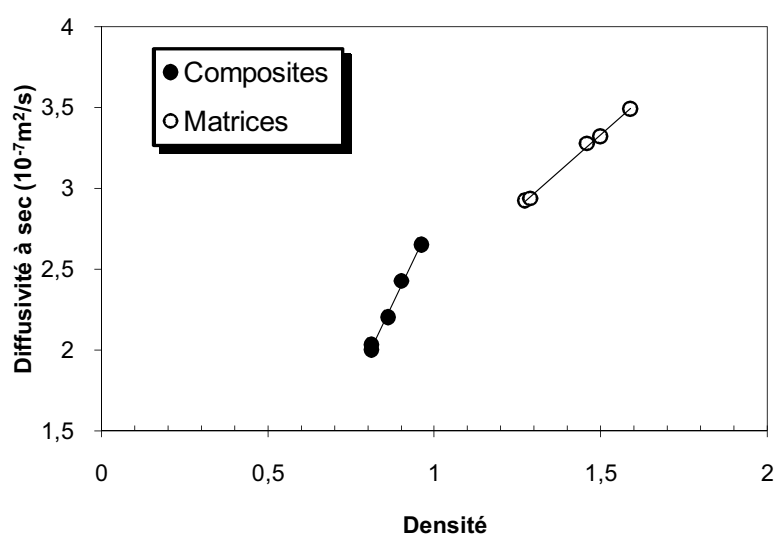

Fig. 2. Evolution de la diffusivité thermique des matériaux utilisés, toutes fines minérales confondues

Pour apprécier les performances thermiques des matériaux élaborés, nous avons rassemblé dans le tableau 9 qui suit, celles de matériaux légers courants.

On peut observer que les matériaux élaborés sont, à densité comparable, des matériaux thermiquement compétitifs. Leurs faibles diffusivités thermiques et leurs hautes capacités calorifiques en font des matériaux intéressants $\mathrm{du}$ point de vue confort et stockage thermiques.

\subsection{Masses volumiques et résistances mécaniques}

Les masses volumiques sèches apparentes ont été déterminées par pesée et mesure des dimensions. d'éprouvettes cubiques de $10 \mathrm{~cm}$ d'arête âgées de 28 jours et séchées à $105^{\circ} \mathrm{C}$ jusqu'à masse constante.
Les résistances à la compression à 28 jours sont obtenues par écrasement de 3 éprouvettes de même géométrie que les précédentes à la vitesse de chargement prévue par la norme NFP 18-406 pour le béton traditionnel. Les résultats sont présentés dans le tableau 10 qui suit.

Il n'existe pas de corrélation entre la résistance à la compression et la masse volumique. A masse volumique égale, les composites confectionnés avec les fines de grès sont 2 fois plus résistants que ceux confectionnés avec les fines d'argile 1. Par ailleurs, dans le cas des fines argileuses, la proportion de quartz ne semble pas jouer un rôle prépondérant. Une étude microstructurelle permettant d'identifier la structure poreuse, la cohésion de la matrice et d'apprécier l'adhérence matrice/granulats sera donc nécessaire.

Remarquons que les bétons de bois élaborés s'apparentent du point de vue mécanique aux bétons légers de classe II (grès et schiste) ou III (granite, $\mathrm{A}_{1}$ et $\mathrm{A}_{2}$ ) de la classification fonctionnelle de la RILEM [13].

Tableau 10: Masse volumique et résistance à la compression des bétons de bois.

\begin{tabular}{|l|c|c|}
\hline $\begin{array}{l}\text { Nature des fines } \\
\text { employées }\end{array}$ & $\begin{array}{c}\text { Masse volumique } \\
\text { apparente sèche }\left(\left(\mathrm{kg} / \mathrm{m}^{3}\right)\right.\end{array}$ & $\begin{array}{c}\mathrm{R}_{\mathrm{c} 28} \text { moyen } \\
(\mathrm{MPa})\end{array}$ \\
\hline Granite & 770 & 3.3 \\
Grès & 730 & 4.6 \\
Schiste & 920 & 4.8 \\
Argile 1 & 740 & 2.0 \\
Argile 2 & 700 & 2.2 \\
\hline
\end{tabular}




\section{Conclusion}

La possibilité de recycler les résidus d'exploitation de carrières par leur transformation en bétons légers d'isolation a été explorée; les résultats expérimentaux obtenus ont montré des qualités intéressantes de leur comportement thermique. La comparaison de leurs performances thermiques avec celles d'autres matériaux légers a prouvé l'intérêt d'un allégement des matrices à base de fines minérales et de ciment portland par des granulats de bois.

Les caractéristiques thermiques à $\mathrm{sec}$ sont intéressantes. Les conductivités thermiques (variant entre 0.117 et $0.190 \mathrm{~W} / \mathrm{m} . \mathrm{K}$ ) témoignent de qualités isolantes sensiblement identiques aux bétons de bois de mêmes densités. Dans le domaine de températures étudiées $(0$ $60^{\circ} \mathrm{C}$ ), les caractéristiques thermiques sont peu sensibles à la variation de la température.

Les fortes valeurs de la capacité calorifique volumique (entre 1.02 et $1.27 \mathrm{MJ} / \mathrm{m}^{3} . \mathrm{K}$ suivant la nature de la fine employée) et les faibles diffusivités thermiques (entre 2 et $2.65 \times 10^{-7} \mathrm{~m}^{2} / \mathrm{s}$ ) en font des matériaux intéressants pour le stockage thermique malgré les faibles densités.

Les matériaux élaborés peuvent trouver des applications en doublage isolant, remplissage et surtout éléments de cloison de partition: blocs, carreaux isolants et même apparents en raison de l'esthétique apporté par la couleur des fines minérales.

\section{Références bibliographiques}

1. Aziz MA, Murphy CK, Ramaswamy SD. International Journal of Lightweight Concretes; 1(1): 29-33. (1979)

2. Salas J, Alvarez M, Veras J. International Journal of Cement Composites and Lightweight Concrete; 8:171-180. (1986)

3. Rahman MA. Materials and Structures; 21: 222227. (1988)

4. Al Rim K, Ledhem A, Douzane O, Dheilly RM, Queneudec M. Cement and Concrete Composites; 14: 341-350. (2000)

5. Bouguerra A. Thèse de Doctorat de l'INSA de Lyon, No d'ordre 97 ISAL 002. (1997)

6. Pimienta P, Chandelier J, Rubaud M, Dutruel F, Nicole H. Cahier du Centre Scientifique et Technique du Bâtiment (CSTB); 2703 (1994)

7. Al Rim K. Thèse de Doctorat de l'université de Rennes I, No. d'ordre 919. (1995)

8. Moslemi AA, Pfister SC. Wood and fiber science; 19(2): 165-175. 1987

9. Perrin B, Javelas R. International journal of heat and mass transfer; 30(2); 297-309. (1987)

10. Laurent JP. International journal of heat and mass transfer; 32(7); 1247-1259. 1989

11. Laurent JP. Matériaux et constructions; 24(141): 221-226. (1991)

12. Gustafsson SE. Review scientific instruments; 62(3): 797-804. (1991)

13. RILEM. Materials and Structures; CPC LC2 11: 61. (1978)
14. Sionneau P, Perier F, Queneudec M, T'kint de Roodenbeke A. Revue Internationale d'héliotechnique; 9: 12-25. (1994)

15. DTU, Document Technique Unifié Règles ThK77:184. (1977)

16. Benmalek ML, Bouguerra A, Goual MS, Bali A, Queneudec M. Creating with Concrete. Exploiting Wastes in Concrete. International Congress, University of Dundee (Scotland).. Thomas Telford Publishing, ISBN 0-7277-28210: 243- 249. (1999)

17. Morabito P. High temperatures-high pressures; 21: 51-59. (1989)

18. Marmoret L. Thèse de Doctorat de l'INSA de Lyon, No. d'ordre 98. (1998) 